\title{
Electronic Predetermination of Ethylene Fragmentation Dynamics
}

\author{
Xinhua Xie, ${ }^{1, *}$ Stefan Roither, ${ }^{1}$ Markus Schöffler, ${ }^{1}$ Erik Lötstedt, ${ }^{2, \$}$ Daniil Kartashov, ${ }^{1}$ Li Zhang, ${ }^{1}$ \\ Gerhard G. Paulus, ${ }^{3,4}$ Atsushi Iwasaki, ${ }^{2}$ Andrius Baltuška, ${ }^{1}$ Kaoru Yamanouchi, ${ }^{2}$ and Markus Kitzler ${ }^{1,}$ \\ ${ }^{1}$ Photonics Institute, Vienna University of Technology, Gusshausstrasse 27, A-1040 Vienna, Austria, EU \\ ${ }^{2}$ Department of Chemistry, School of Science, The University of Tokyo, \\ 7-3-1 Hongo, Bunkyo-ku, Tokyo 113-0033, Japan \\ ${ }^{3}$ Institute of Optics and Quantum Electronics, Friedrich-Schiller-University Jena, \\ D-07743 Jena, Germany, EU \\ ${ }^{4}$ Helmholtz Institute Jena, D-07743 Jena, Germany, EU
}

(Received 14 October 2013; published 7 April 2014)

\begin{abstract}
We experimentally investigate the dependence of the fragmentation behavior of the ethylene dication on the intensity and duration of the laser pulses that initiate the fragmentation dynamics by strong-field double ionization. Using coincidence momentum imaging for the detection of ionic fragments, we disentangle the different contributions of ionization from lower-valence orbitals and field-driven excitation dynamics to the population of certain dissociative excited ionic states that are connected to one of several possible fragmentation pathways towards a given set of fragment ions. We find that the excitation probability to a particular excited state and therewith the outcome of the fragmentation reaction strongly depend on the parameters of the laser pulse. This, in turn, opens up new possibilities for controlling the outcome of fragmentation reactions of polyatomic molecules in that it may allow one to selectively enhance or suppress individual fragmentation channels, which was not possible in previous attempts of controlling fragmentation processes of polyatomic molecules with strong laser fields.
\end{abstract}

DOI: 10.1103/PhysRevX.4.021005

\section{INTRODUCTION}

The fragmentation of a polyatomic molecule, potentially preceded by isomerization processes such as proton migration [1], is of fundamental importance in nature. These nuclear dynamical processes take place on multidimensional potential energy surfaces and are driven by the derivatives of these surfaces along certain reaction coordinates. Before any nuclear motion sets in, however, much faster electronic processes may take place. A fascinating question is to what extent and by which mechanisms electronic processes can dynamically influence or determine the outcome of the slower isomerization and fragmentation processes. Strong laser fields can be used to both initiate and drive electronic dynamics on their natural, i.e., subfemtosecond, time scale. This potentially opens up the feasibility of controlling molecular fragmentation processes by influencing the intramolecular dynamics of the

\footnotetext{
*xinhua.xie@tuwien.ac.at

†markus.kitzler@tuwien.ac.at

"Present address: Laser Technology Laboratory, RIKEN, 2-1 Hirosawa, Wako, Saitama 351-0198, Japan.

Published by the American Physical Society under the terms of the Creative Commons Attribution 3.0 License. Further distribution of this work must maintain attribution to the author(s) and the published article's title, journal citation, and DOI.
}

Subject Areas: Atomic and Molecular Physics, Optics, Physical Chemistry electron cloud with strong laser-electric fields. Indeed, it has been shown that the outcome of fragmentation processes that start from ionic states of $\mathrm{H}_{2} / \mathrm{D}_{2}$ and $\mathrm{CO}$ can be affected by the shape of the laser electric field (see Refs. [2-5] and references therein). Recently, this type of strong-field control of molecular fragmentation has been further extended to polyatomic molecules [6].

In general, the key to controlling fragmentation and accompanying isomerization processes in molecular ions is to prepare them in specific dissociative states from which the reaction proceeds through a desired fragmentation pathway on multidimensional potential energy surfaces towards a certain set of final fragment products, called a channel. This can be achieved either during the ionization process or by subsequent excitation processes during the interaction with the laser field. The preparation of the molecule in a specific dissociative state during the ultrashort interaction of the laser pulse with the molecule, thus, predetermines the potentially much slower isomerization and fragmentation dynamics that can take place long after the laser pulse has faded. Preparation of a molecular ion in a certain dissociative excited state by ionization is possible by removing specific inner-valence shell electrons [6-8]. It has been shown that this type of preparation can be used to predetermine molecular fragmentation on a laser-subcycle time scale [6]. Excitation to higher ionic energy levels during the laser pulse, on the other hand, can be achieved 
by field-induced transitions [9-12] or by electron recollision [13-15]. In practice, a mixture of these excitation mechanisms may be at work and their relative importance may strongly depend on the laser pulse parameters, in particular, on intensity and pulse duration [16,17]. In order to extend light-field control of molecular fragmentation to larger and more complex polyatomic molecules where a variety of different dissociation pathways coexist, it is necessary to enhance our knowledge about the coupling between the fast field-induced electronic dynamics and the resulting slower nuclear motion, and about the dependence of these processes on the laser parameters.

Here, we report experiments on the polyatomic molecule ethylene $\mathrm{C}_{2} \mathrm{H}_{4}$ dedicated to disentangling the contributions of the ionization step and the subsequent field-driven excitation dynamics to different fragmentation pathways from the doubly charged ion. In particular, we show how these two excitation mechanisms that predetermine the fragmentation reactions of the molecule depend on laser pulse intensity and duration. To this end, the experiments employ pulses with durations ranging from the few-cycle regime to $25 \mathrm{fs}$ and intensities from $5 \times 10^{14}$ to $2 \times 10^{15} \mathrm{~W} / \mathrm{cm}^{2}$. In this intensity range, double ionization is dominated by a sequential dynamics, where two electrons are removed one after another [18-20]. Coincidence detection of the resulting fragment ions by ion momentum imaging allows the reconstruction of the fragmentation pathways along different dissociative excited ionic states.

The fragmentation of polyatomic molecules induced by laser pulses has been the subject of extensive research for decades. Hydrocarbons with their intrinsically very fast intramolecular nuclear dynamics of the light hydrogen atoms or protons, which leads to a rich restructuring dynamics, are particularly interesting and have been the object of numerous experimental studies, both using coincidence detection of the molecular fragments $[1,6-8,16,17,21-25]$ and without coincidence detection $[9,10,26,27]$.

In the present work, we observe that the relative importance of contributions to the fragmentation probability of a given channel, either from the ionization step or from field-driven excitations, strongly depends on both laser intensity and pulse duration. Moreover, we show that not only the probability of a given channel, but even the specific pathways that can be taken along the multitude of dissociative electronic states towards this channel, are dependent on the laser pulse parameters. Thus, by properly choosing pulse intensity and duration, it becomes possible to steer the molecular dynamics along a desired pathway in the phase space spanned by the nuclear coordinates and momenta towards a certain set of final fragment ions. This opens up new possibilities for controlling the outcome of fragmentation reactions of polyatomic molecules in that it may allow one to selectively enhance or suppress individual fragmentation channels, which was not possible in previous attempts of controlling the fragmentation behavior of polyatomic molecules [6].

\section{EXPERIMENTS}

In our experiments, we measured in coincidence the three-dimensional (3D) momentum vectors of fragment ions resulting from the interaction of a laser pulse with an ethylene molecule using a cold target recoil ion momentum spectroscopy (COLTRIMS) setup [28,29]. Details of the experimental setup can be found in our previous publications $[16,25,30]$. In short, the laser beam, linearly polarized along the spectrometer axis, was focused in an ultrahigh vacuum chamber $\left(\sim 1.3 \times 10^{-10}\right.$ mbar $)$ by a spherical $\mathrm{Ag}$ mirror with $60 \mathrm{~mm}$ focal length onto a supersonic gas jet ( $\sim 170 \mu \mathrm{m}$ in diameter) of ethylene molecules. The resulting ions were guided to a time- and position-sensitive channel-plate detector by a weak homogeneous electric field $(23 \mathrm{~V} / \mathrm{cm})$. In the measurement, the momentum resolution of ions along the laser polarization direction ( $z$ axis) was about 1 a.u., while that along the jet direction ( $x$ axis) and that along the laser propagation direction ( $y$ axis) was about 4 and 2 a.u., respectively. Fewcycle laser pulses were generated at a repetition rate of $5 \mathrm{kHz}$ by spectral broadening of $25 \mathrm{fs}$ (FWHM) laser pulses with a spectrum centered around $795 \mathrm{~nm}$ from a Ti:sapphire chirped-pulse laser amplifier system in a 1-m-long hollow-core glass capillary filled with neon at a pressure of several atmospheres. Temporal recompression of the pulses after the capillary by several bounces from pairs of chirped mirrors and subsequently passing them through a pair of glass wedges for fine-tuning the dispersion results in a pulse duration of $\sim 4.5 \mathrm{fs}$. The duration and intensity stability of the pulses were monitored on a shot-to-shot basis by a stereo-above-threshold-ionization phase meter $[31,32]$. The pulse duration was varied between 4.5 and 17 fs by positively chirping the shortest pulses by propagating them through different amounts of fused silica. Pulses with a duration of $25 \mathrm{fs}$ were used directly from the chirped pulse amplifier system. The peak intensity of the pulses in the measurement vacuum chamber was varied from $2 \times 10^{14}$ up to $\sim 2 \times 10^{15} \mathrm{~W} / \mathrm{cm}^{2}$ by reflecting the beam off a thick fused silica block under different angles, thereby changing the pulse energy. Calibration of the peak intensity on target was done with an estimated precision of $\pm 10 \%$ by separate measurements using single ionization of argon atoms in circularly polarized light [33].

\section{RESULTS AND DISCUSSION}

Ethylene is a planar molecule, and the electron configuration of its neutral state is $\left(1 a_{g}\right)^{2}\left(1 b_{1 u}\right)^{2}\left(2 a_{g}\right)^{2}\left(2 b_{1 u}\right)^{2}$ $\left(1 b_{2 u}\right)^{2}\left(3 a_{g}\right)^{2}\left(1 b_{3 g}\right)^{2}\left(1 b_{3 u}\right)^{2}$. As illustrated in Fig. 1(a), the electron configuration of the HOMO $\left(1 b_{3 u}\right)$ orbital is a $\pi$ state whose electron density is dominantly distributed perpendicular to the molecular plane, whereas that of the HOMO-1 $\left(1 b_{3 g}\right)$ orbital is mainly distributed along the $\mathrm{C}-\mathrm{H}$ bond directions and that of the HOMO-2 $\left(3 a_{g}\right)$ orbital along the $\mathrm{C}-\mathrm{C}$ bond. The vertical ionization potentials of 


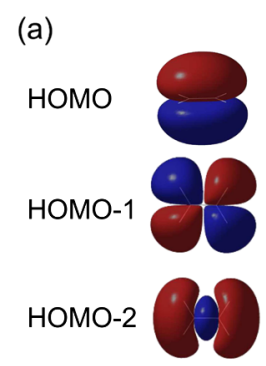

(b)

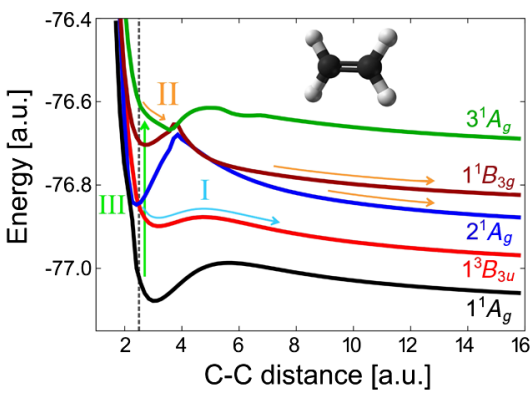

FIG. 1. (a) Molecular orbitals of ethylene calculated by GAMESS [37] as described in the text. (b) Calculated PESs of ground $\left(1^{1} A_{g}\right.$, black line) and selected excited ionic states of the ethylene dication. The ${ }^{3} B_{3 u}$ state is formed by removing one HOMO electron and one HOMO-2 electron, the ${ }^{1} B_{3 g}$ by removing one HOMO- 1 electron and one HOMO-2 electron, and the $2^{1} A_{g}$ and $3^{1} A_{g}$ states are formed by removing two HOMO-1 electrons and two HOMO-2 electrons, respectively. The vertical black dashed line indicates the C-C equilibrium distance of 2.53 a.u. The colored arrows (labeled by I, II, and III) refer to the three fragmentation pathways of channel (1), as discussed in the text.

removing one electron from these orbitals were predicted to be $10.8 \mathrm{eV}$ (HOMO), $13.2 \mathrm{eV}$ (HOMO-1), and $15.0 \mathrm{eV}$ (HOMO-2) [34]. The double ionization potential of ethylene into the ground ionic state of the ethylene dication is $29.46 \mathrm{eV}$ [35].

Nonsequential double ionization of hydrocarbon molecules in a laser field was previously investigated by mass spectroscopy, and it was found to be the dominating process when the laser intensity is below a few $10^{14} \mathrm{~W} / \mathrm{cm}^{2}$ [18-20]. The Keldysh parameter [36] indicates that the double ionization step takes place dominantly via tunneling when the laser peak intensity approaches $5 \times 10^{14} \mathrm{~W} / \mathrm{cm}^{2}$. In our experiments, we use intensities equal to or higher than $5 \times 10^{14} \mathrm{~W} / \mathrm{cm}^{2}$, which places us in the regime where double ionization dominantly happens sequentially.

Earlier experiments with EUV radiation as well as theoretical studies found that double ionization of ethylene involving the removal of electrons from lower-lying orbitals leads to the breaking of the central C-C or of one of the $\mathrm{C}-\mathrm{H}$ bonds $[35,38,39]$. By a coincidence analysis of the measured data recorded during our experiments that comprises the application of momentum conservation conditions in all three spatial dimensions, we accordingly identified for all pulse parameters the following two-body fragmentation channels of the ethylene dication:

$$
\begin{aligned}
& \mathrm{C}_{2} \mathrm{H}_{4}^{2+} \rightarrow \mathrm{CH}_{2}^{+}+\mathrm{CH}_{2}^{+}, \\
& \mathrm{C}_{2} \mathrm{H}_{4}^{2+} \rightarrow \mathrm{C}_{2} \mathrm{H}_{3}^{+}+\mathrm{H}^{+} .
\end{aligned}
$$

In addition, we identified the channel

$$
\mathrm{C}_{2} \mathrm{H}_{4}^{2+} \rightarrow \mathrm{C}_{2} \mathrm{H}_{2}^{+}+\mathrm{H}_{2}^{+} \text {. }
$$

We found that channel (3) shows similar momentum distributions as channel (2), which indicates that it features similar fragmentation dynamics. In the following, we will, therefore, restrict ourselves to discussing the fragmentation dynamics of the channels (1) and (2).

As discussed above, population of a dissociative excited state in the dication, and therewith initiation of a fragmentation reaction, can be achieved both by ionization from lower-valence orbitals and by field-driven excitations taking place after or in the course of the ionization dynamics. In the following, we first focus on identifying the contributions of ionization from lower-valence shells to the fragmentations through channels (1) and (2), and on discussing their dependence on the laser pulse parameters. Subsequently, we discuss the influence of field-driven excitations.

\section{A. Fragmentations induced by lower-valence shell ionization}

It has been shown for small (diatomic and triatomic) molecules that strong-field ionization may not only take place from the HOMO, but that during the ionization process, electron density is also removed from the HOMO1 and HOMO-2 [40-44]. As compared to diatomic and triatomic molecules, the energy gaps between molecular orbitals are considerably smaller in polyatomic hydrocarbons. Thus, the contributions from lower-valence orbitals are expected to be more pronounced for ionization of hydrocarbons. In addition, as the nuclear motion in hydrocarbons exhibits many more degrees of freedom, a much richer and more complicated electronic and nuclear dynamics may be expected during (and after) the interaction with the laser pulse.

Figures 2(a)-2(d) compare measured momentum distributions of fragment ions from channels (1) and (2) for 4.5 and $25 \mathrm{fs}$ pulses at an intensity of $5 \times 10^{14} \mathrm{~W} / \mathrm{cm}^{2}$. A clear observation is that the angular momentum distribution of channel (1) is anisotropic and peaks along the laser polarization direction, whereas that of channel (2) is isotropic. This can be seen even more clearly in the normalized angular momentum distributions shown in Fig. 2(e). The angular momentum distribution of channel (3) was also analyzed and was found to be isotropic, similar to that of channel (2). In our experiments, we also observe a considerable number of hits from the ethylene dication. This is because the lifetime of the metastable ground state of the ethylene dication [see Fig. 1(b)], formed by removing two electrons from the HOMO, is sufficiently large such that $\mathrm{C}_{2} \mathrm{H}_{4}{ }^{2+}$ ions survive the flight time to the detector with a high probability. Removal of electrons from lowerlying molecular orbitals, on the other hand, will directly put the dication into an electronically excited dissociative state, which will lead to fast fragmentations of the dication. 


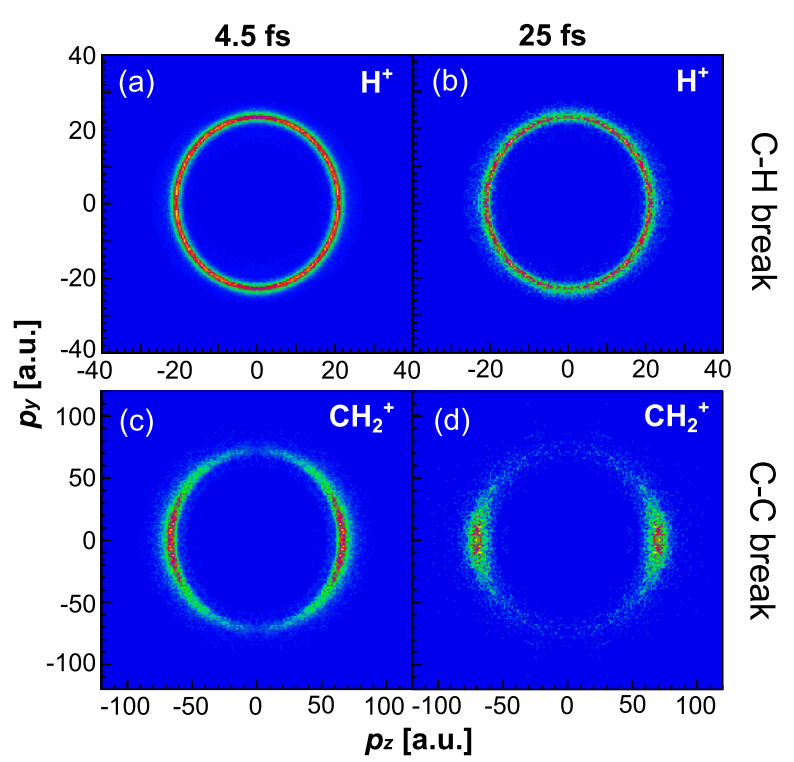

(e)

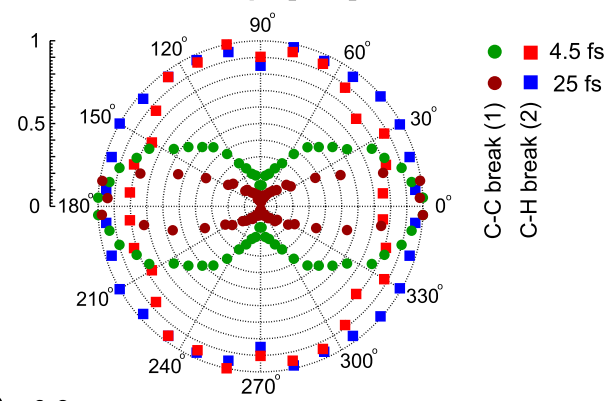

(f)

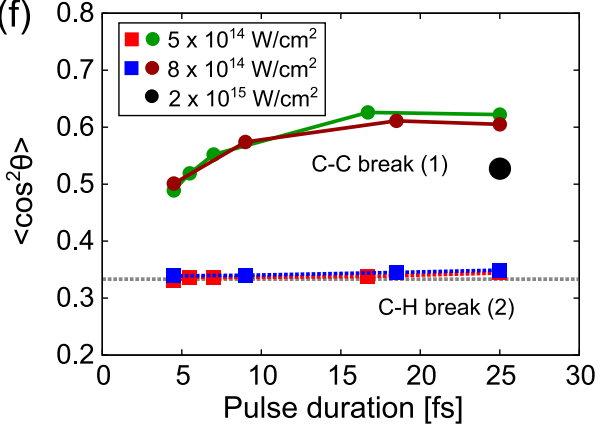

FIG. 2. (a)-(d) Slices through the 3 D momentum distributions in the $y z$ plane $\left(\left|p_{x}\right|<5\right.$ a.u.) for the indicated fragments from channels (2) (a),(b) and (1) (c),(d). Pulse duration 4.5 fs (left) and $25 \mathrm{fs}$ (right), intensity $5 \times 10^{14} \mathrm{~W} / \mathrm{cm}^{2}$ for each panel, polarization direction horizontal (z). (e) Normalized angular momentum distributions for channels (1) and (2). Pulse parameters as indicated. Polarization direction horizontal. (f) $\left\langle\cos ^{2} \theta\right\rangle$ over pulse duration for channels (1) and (2). Peak intensity as indicated.

The ionization rate from a certain orbital strongly depends on the relative orientation of the molecular orbital to the laser polarization direction and follows the shape of the electron density distribution of the respective orbital $[45,46]$. The angle-dependent ionization rate for removing a HOMO-2 electron thus peaks along the laser polarization direction and parallel to the $\mathrm{C}-\mathrm{C}$ axis, while that for removing a HOMO electron peaks perpendicular to the
$\mathrm{C}-\mathrm{C}$ axis. As a consequence, the anisotropic angular momentum distribution of channel (1) with peaks along the laser polarization direction indicates, first, that the fragmentation process takes place from an excited ionic state of the ethylene dication formed by ionization of at least one HOMO-2 electron. Second, the strong anisotropy indicates that the fragmentation happens fast as compared to the rotational period of $\mathrm{C}_{2} \mathrm{H}_{4}{ }^{2+}$ [21]. The isotropic angular momentum distribution of channel (2) [and also that of channel (3)], in contrast, suggests that the separation of the two fragments takes longer than the rotational period and happens long after the laser pulse has faded [35,47]. The fragmentation dynamics of channels (1) and (2), thus, take place apparently on different time scales.

Our experimental results can be qualitatively explained by the potential energy surfaces of excited ionic states of the ethylene dication calculated by the quantum chemistry simulation package GAMESS [37]. The excited states were calculated by a full configuration interaction option, but restricting the number of active electrons and orbitals. Twodimensional potential energy surfaces (PESs) of the neutral and dication were calculated as a function of the $\mathrm{C}-\mathrm{C}$ distance and of one of the $\mathrm{C}-\mathrm{H}$ distances. The ground state of the ethylene dication (reached by removing two HOMO electrons) is found to be a metastable state, which explains the observation of the ethylene dication. Cuts through the PESs along the C-C distance of several electronically excited states of the ethylene dication are shown in Fig. 1(b). These excited states can be reached directly by the removal of one or two electrons from lower lying molecular orbitals, as described in the figure caption. Cuts of the PESs along the C-H distance (not shown) reveal that the lowest electronically excited state that may lead to C-H breaking, induced by removal of one HOMO-1 electron and one HOMO electron, shows a shallow but thick barrier along the $\mathrm{C}-\mathrm{H}$ distance. Therefore, this state exhibits a long lifetime, and dissociation of the ethylene dication along the $\mathrm{C}-\mathrm{H}$ bond is slow, in agreement with the experimental finding of an isotropic angular distribution of $\mathrm{H}^{+}$ions discussed above. On the other hand, the simulated PESs reveal that the lowest electronically excited state that leads to $\mathrm{C}-\mathrm{C}$ bond breaking is the ${ }^{3} B_{3 u}$ state, which is formed by removing one HOMO-2 electron together with one HOMO electron, as shown in Fig. 1(b). Since the electron density of HOMO-2 is mainly distributed along the $\mathrm{C}-\mathrm{C}$ axis, because of the angular sensitivity of the ionization rate to the electron density distribution, among the randomly oriented molecules in the gas sample, those molecules with their $\mathrm{C}-\mathrm{C}$ axis aligned with the laser polarization direction are much more likely to become doubly ionized through removal of at least one HOMO-2 electron. This explains the anisotropic angular momentum distribution of channel (1) observed in the experiment.

As a cross-check for our interpretation of the measured angular distributions of the fragmentation yields, we 
performed additional measurements with different laser peak intensities and pulse durations. Figure 2(f) shows the anisotropy of the angular distributions of the two channels (1) and (2), characterized by the value $\left\langle\cos ^{2} \theta\right\rangle$, as a function of pulse duration for different laser intensities. Here, $\theta$ is defined as the angle between the ion momentum vector and the laser polarization direction. It can be clearly seen that the angular momentum distribution of channel (2) has no dependence on either laser peak intensity or pulse duration and stays isotropic $\left(\left\langle\cos ^{2} \theta\right\rangle=1 / 3\right)$ for all pulse parameters. This further confirms our explanation that the fragmentation happens long after the laser pulse has faded, with enough time for molecular rearrangement after the double ionization. For channel (1), however, $\left\langle\cos ^{2} \theta\right\rangle$ increases with increasing laser pulse duration. This can be explained by postionization alignment [48]. With increasing laser pulse duration, the effect of the postionization alignment becomes stronger, which, in turn, leads to more anisotropic angular distributions and higher values of $\left\langle\cos ^{2} \theta\right\rangle$.

\section{B. Influence of field-induced excitations}

Now, we turn to study the importance of field-induced excitation dynamics during and after double ionization of ethylene. One additional observation for channel (1) is that the value of $\left\langle\cos ^{2} \theta\right\rangle$ decreases from 0.61 to 0.52 with increasing laser intensity from $8 \times 10^{14}$ to $2 \times 10^{15} \mathrm{~W} / \mathrm{cm}^{2}$, which contradicts the general trend expected for postionization alignment [see Fig. 2(f)]. With higher laser intensity, the postionization alignment effect should be stronger, which, in turn, should lead to a larger $\left\langle\cos ^{2} \theta\right\rangle$. To obtain insight into the reasons for this observation, we analyze the kinetic energy release (KER) spectrum of channel (1) for different intensities and pulse durations. Figure 3(a) shows KER spectra of channel (1) for three different laser intensities but the same pulse duration of $25 \mathrm{fs}$. It can be clearly seen that with increasing laser intensity the mean value of the KER of the fragmentation increases from around $5 \mathrm{eV}$ up to $7 \mathrm{eV}$. Additional measurements for smaller intensities reveal that, in the range of 2 to $5 \times 10^{14} \mathrm{~W} / \mathrm{cm}^{2}$, the KER stays at $5 \mathrm{eV}$ and is largely independent of the laser intensity and pulse duration. However, when the laser intensity is as high as $8 \times 10^{14} \mathrm{~W} / \mathrm{cm}^{2}$ or above, the KER of channel (1) increases with increasing laser intensity. In contrast, the KER values of the fragmentation channels (2) and (3) have a mean value of about $4 \mathrm{eV}$ and were found to be independent of the laser intensity and pulse duration.

The dependence of the KER of fragmentation channel (1) on laser intensity indicates that the fragmentation of the $\mathrm{C}-\mathrm{C}$ bond takes place via different pathways for different laser pulse parameters. As discussed above, this may involve the population of different initial dissociative (excited) states by ionization from different lower-valence orbitals and/or field-induced excitations to other excited
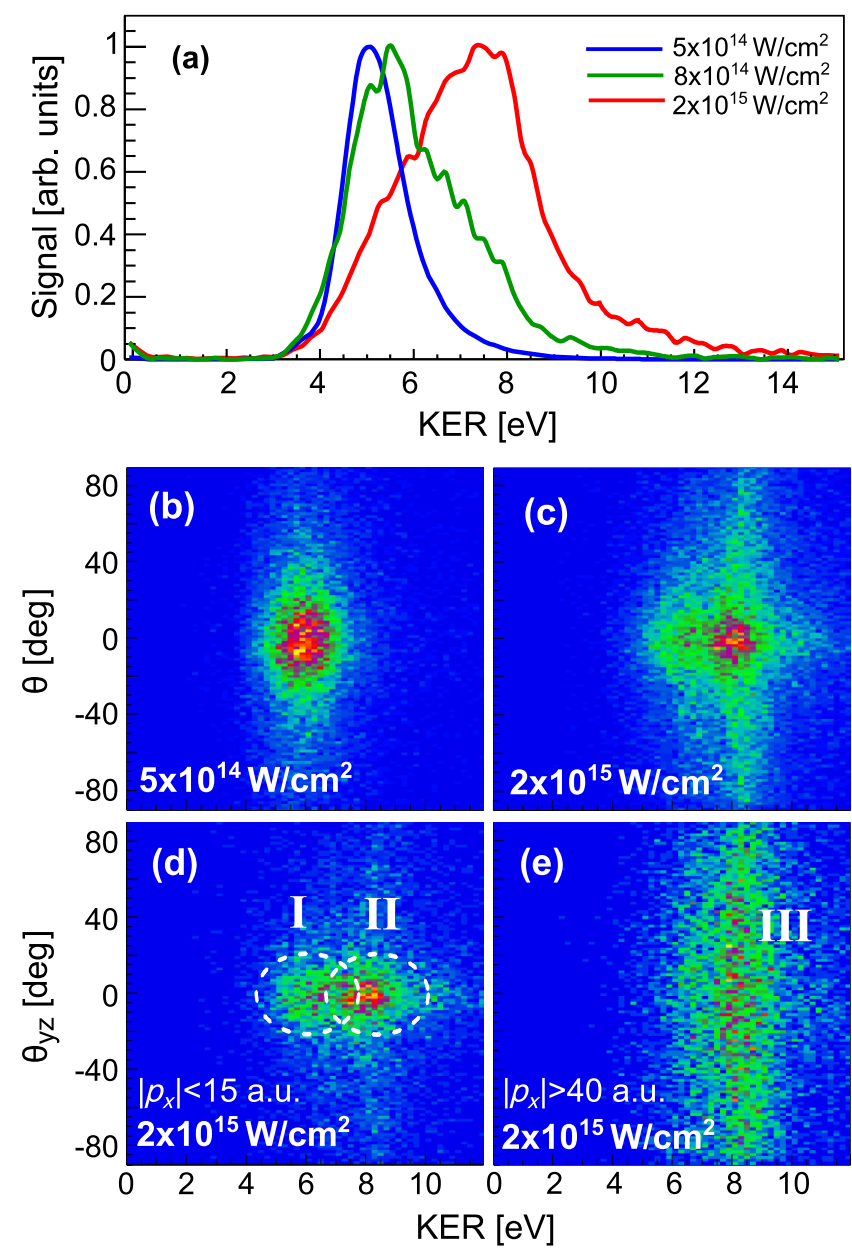

FIG. 3. (a) Normalized KER spectra of fragmentation channel (1) for three different laser intensities as indicated. (b)-(e) Angular distributions over KER of $\mathrm{CH}_{2}^{+}$fragments from channel (1) measured at the indicated laser intensities. (d),(e) Selections of the angular distribution shown in (c) for $\left|p_{x}\right|<15$ a.u. (d) and $\left|p_{x}\right|>40$ a.u. (e) in a plane parallel to the $y z$ plane, with $\theta_{y z}$ the angle to the $z$ axis in this plane. See text for details.

states by the action of the laser pulse. In the following, we demonstrate that a combination of coincidence selection in both energy and angular direction, i.e., in KER and $\theta$, enables us to uniquely identify the fragmentation dynamics and therewith disentangling different contributions to the overall fragmentation yield. As we show, breaking of the $\mathrm{C}-\mathrm{C}$ bond can take place via three different pathways starting from dissociative excited states populated by ionization from different lower-valence orbitals as well as by field-induced excitations.

Figures 3(b) and 3(c) show KER spectra as a function of $\theta$ for $5 \times 10^{14}$ and $2 \times 10^{15} \mathrm{~W} / \mathrm{cm}^{2}$, respectively. The opening of new pathways for the higher intensity becomes apparent by the additional contributions at higher KER and larger $\theta$. The first step in disentangling the different pathways is to identify the (lower-valence) orbitals from which electrons are removed during ionization. As was 
shown above, this information is encoded in the angular distributions of the fragments. Thus, selection along the angular direction can provide us with information about the ionization process. Figures 3(d) and 3(e) show angular distributions of channel (1) in a plane parallel the $y z$ plane, measured at a laser intensity of $2 \times 10^{15} \mathrm{~W} / \mathrm{cm}^{2}$, for two different selections along $\left|p_{x}\right|$. Cutting a slice from the momentum sphere that contains the $z$ axis, $\left|p_{x}\right|<15$ a.u., enhances the relative contributions of events that peak along the laser polarization direction $z$; see Fig. 3(d). Selecting a shell from the momentum sphere sufficiently far away from its center, $\left|p_{x}\right|>40$ a.u., cuts away the contributions that peak along the laser polarization direction; see Fig. 3(e). Three regions (marked I, II, and III in the figures) can be clearly distinguished. Regions I and II with a KER around 5 and $8 \mathrm{eV}$, respectively, both with narrow angular distributions confined to close the laser polarization direction, show up in the $\left|p_{x}\right|<15$ a.u. selection presented in Fig. 3(d). Region III, visible in the selection $\left|p_{x}\right|>$ 40 a.u. presented in Fig. 3(e), has the same KER around $8 \mathrm{eV}$ as region II but exhibits isotropic angular distribution.

First, we focus on the narrow angular distributions. As was shown above, a narrow angular distribution (with large $\left\langle\cos ^{2} \theta\right\rangle$ ) peaking along the laser polarization direction suggests the removal of at least one HOMO-2 electron. Removal of one HOMO-2 electron and one HOMO electron prepares the dication in the $1 B_{3 u}$ state. This is the lowest ionic state that can be reached by removing one HOMO-2 electron, and is dissociative along the C-C direction, as marked by the cyan arrow in Fig. 1(b). The KER of this pathway is estimated from the theoretical potential energy curves as about $5 \mathrm{eV}$, in very good agreement with the measured KER of region I. Thus, theory confirms that the fragmentation events in region I originate from a pathway along a dissociative excited state that is populated by removing an electron from both the HOMO and the HOMO-2.

Removal of two electrons from the HOMO-2 can put the ethylene dication onto the second lowest electronically excited state that leads to the breaking of the $\mathrm{C}-\mathrm{C}$ bond $\left(3^{1} A_{g}\right)$. From this state, it will dissociate through crossings with the $2^{1} A_{g}$ and ${ }^{1} B_{3 g}$ states [Fig. 1(b)]. As the initial state is populated by ionization from the HOMO-2, the angular distribution of the fragment ions will still be narrow along the laser polarization direction. However, fragmentations along this pathway II [marked by orange arrows in Fig. 1(b)] will lead to a higher KER. Thus, the additional information on the ionization step provided by the KER in addition to the angular distribution allows us to distinguish the initial population of different excited dissociative states. Theory predicts values of 8 and $9 \mathrm{eV}$, respectively, for dissociation along the ${ }^{1} B_{3 g}$ and $2^{1} A_{g}$ states, which fits the measured KER energy range for recorded events in region II very well [Fig. 3(d)].
We now turn to the fragments in region III [Fig. 3(e)]. Their KER distribution is located around $8 \mathrm{eV}$ (similarly to that of the fragmentations in pathway II), but their angular distribution is isotropic, which is a marked difference from pathway II. The isotropic angular distribution indicates that the fragmentation starts from an excited ionic state formed by removal of electrons from the HOMO or HOMO-1. This would prepare the ion into the metastable ground state or a low excited state, which will not lead to breaking of the C-C center bond. Thus, the pathway leading to the fragment ions in region III must involve a field-induced excitation to the higher excited state $3^{1} A_{g}$ induced by the laser pulse. From there, it dissociates along the same PESs as pathway II, leading to fragments with almost the same high KER as pathway II.

Using the combined information provided by the KER and the angular distribution of the fragment ions, permitted us to distinguish the three different pathways contributing to channel (1). We show above that these pathways are initiated by the removal of electrons from different lowerlying valence orbitals as well as by additional field-induced population transfer to higher-lying excited states. A crucial laser pulse parameter that controls the relative yields of these three different pathways is the peak intensity. When the intensity is $5 \times 10^{14} \mathrm{~W} / \mathrm{cm}^{2}$ or below, only pathway I exists. With increasing intensity, the additional pathways II and III are opened up [Fig. 3], as the ionization probability from low valence shells (in our case, removal of a second electron from the HOMO-2) increases, and field-induced excitations to higher excited states become possible. As a consequence, by adjustment of the laser pulse parameters, selective control of the fragmentation channel and possibly even the pathway by predetermining the dissociation of a polyatomic molecule on the electronic level via preparation in a specific electronically excited dissociative state becomes feasible. A practical implementation of this type of laser pulse control of fragmentation is discussed in the following section.

\section{Electronic predetermination of fragmentation pathways}

To demonstrate the selective fragmentation of a polyatomic molecule for the example of ethylene, we consider the ratio between channels (1) and (2), i.e., the ratio between fragmentation along the $\mathrm{C}-\mathrm{C}$ and $\mathrm{C}-\mathrm{H}$ bonds, respectively. This ratio is shown in Fig. 4(a) over pulse intensity. For low laser intensities, the relative probability of fragmentation along the $\mathrm{C}-\mathrm{C}$ bond is small and fragmentations take place dominantly via breaking of a $\mathrm{C}-\mathrm{H}$ bond. When increasing the laser peak intensity, the relative probability of fragmentations along the $\mathrm{C}-\mathrm{C}$ bond strongly increases and becomes equally large to that of $\mathrm{C}-\mathrm{H}$ breaking at an intensity of $2 \times 10^{15} \mathrm{~W} / \mathrm{cm}^{2}$. As discussed above, this is because, for the higher peak intensities, new pathways from higher-lying electronically excited states of 
the dication (leading to higher KER) are opened up for the fragmentation along channel (1), while the fragmentation along channel (2) does not necessitate the removal of electrons from low-lying valence orbitals (and therewith no high intensities) nor is it possible to influence the slow dissociation dynamics of channel (2) via field-induced population transfer during the short laser pulse.

Finally, we consider using the pulse duration as a parameter to control the fragmentation dynamics from the ethylene dication. Clearly, both the ionization probability from lower-valence orbitals as well as the probability of inducing field-induced excitations should depend on the duration of the laser pulse. Figure 4(b) shows the ratio of counts resulting from the fragment ions to those resulting from the parent ion $\mathrm{C}_{2} \mathrm{H}_{4}^{2+}$ for peak intensities $5 \times 10^{14}$ and $8 \times 10^{14} \mathrm{~W} / \mathrm{cm}^{2}$. For short pulse durations, the yield of fragment ions exceeds the parent ion yield; i.e., the probability of preparing the molecule in an excited dissociative state is higher than preparing it in the ground state. However, when the pulse duration increases from 4.5 to $9 \mathrm{fs}$, the ratio strongly decreases and becomes independent of the pulse duration for longer pulses. A possible interpretation for this somewhat counterintuitive behavior is that

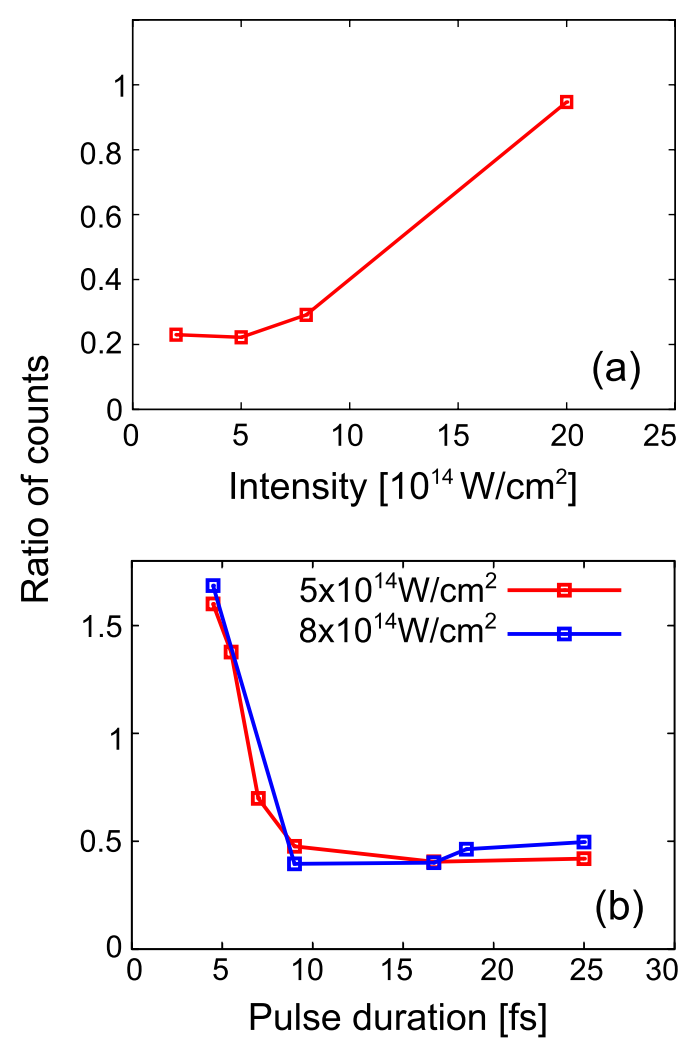

FIG. 4. (a) Ratio of event numbers for channel (1) with respect to those for channel (2) measured for a pulse duration of $25 \mathrm{fs}$ as a function of laser peak intensity. (b) Sum of event numbers in channels (1) and (2) normalized to the detected counts of the parent ion $\mathrm{C}_{2} \mathrm{H}_{4}^{2+}$ over laser pulse duration for the two peak intensities $5 \times 10^{14} \mathrm{~W} / \mathrm{cm}^{2}$ (red) and $8 \times 10^{14} \mathrm{~W} / \mathrm{cm}^{2}$ (blue). longer laser pulses depopulate the doubly charged states due to enhanced ionization [25,49-51] to higher charge states. The pulse duration of $9 \mathrm{fs}$, where saturation levels off, is thus related to the time that the $\mathrm{C}-\mathrm{H}$ bonds need to stretch from their equilibrium distance to the critical internuclear distance, in good agreement with the $\mathrm{C}-\mathrm{H}$ bond vibrational period of about $10 \mathrm{fs}$. Details of the pulse duration dependence of enhanced ionization of ethylene in strong laser fields can be found in Ref. [52].

Importantly, however, the result shown in Fig. 4(b) highlights that the population of electronically excited states (due to both lower-valence ionization and fielddriven excitation) during the interaction of a polyatomic molecule with an intense nonresonant laser pulse is not a negligible process [8], even for the shortest laser pulses. As the population of excited states initiates potentially very fast molecular restructuring dynamics (such as $\mathrm{C}-\mathrm{H}$ stretch motion considered here), it must be considered for any investigation of polyatomic molecules concerned with probing structure using intense laser pulses, including high-harmonic generation [53], laser-induced electron self-diffraction [54], and particularly in the retrieval of geometric information [22].

\section{CONCLUSION AND OUTLOOK}

In conclusion, we investigate the dependence of the fragmentation dynamics of different fragmentation channels from the ethylene dication on laser pulse intensity and duration. By the analysis of the KER and the angular distribution of fragment ions measured by coincidence momentum imaging, we were able to disentangle the different contributions of (i) ionization from lower-valence orbitals and (ii) field-induced population transfer to the overall yield of the different fragmentation channels. We demonstrate that the relative importance of the different molecular pathways along different dissociative electronically excited states, by which a particular set of final fragmentation products can be reached, strongly depends on the parameters of the laser pulse. Our work shows that selective population of excited ionic states by controlling intramolecular electronic processes (in particular, electron removal from lower-valence orbitals and nonadiabatic population transfer) with strong nonresonant laser fields is an efficient and general method for selectively enhancing or suppressing individual fragmentation channels. In this work, we demonstrate a selective preparation of the molecular ion in a certain excited state by exploiting the sensitivity of lower-valence electron removal on the intensity of the laser pulse. Application of this scheme for fragmentation control is, however, by no means restricted to the parameters intensity and duration that we use in this work, but can straightforwardly be combined with the carrier-envelope offset phase $[2,6]$. Probably even more important, though - as ionization from certain (lowervalence) orbitals can be enhanced (or suppressed) for a 
given orientation of the molecule with respect to the laser polarization direction - in this scheme laser alignment or orientation of the molecule $[55,56]$ can serve as a particularly effective control parameter for the selective population of certain dissociative excited ionic states and for obtaining an enhanced channel selectivity. Experiments that exploit this opportunity are under way.

\section{ACKNOWLEDGMENTS}

We acknowledge financial support from the Austrian Science Fund (FWF), Grants No. P21463-N22 and No. P25615-N27, a Starting Grant from the European Research Council (ERC), project $\mathrm{CyFi}$, the Japanese Society for the Promotion of Science (JSPS) (09035011000061), the Ministry of Education, Culture, Sports, Science, and Technology (MEXT), Japan, the grant-inaid for Specially Promoted Research on Ultrafast Hydrogen Migration (19002006), and the grant-in-aid for Global COE Program for Chemistry Innovation.

[1] H. Xu, T. Okino, K. Nakai, K. Yamanouchi, S. Roither, X. Xie, D. Kartashov, M. Schöffler, A. Baltuska, and M. Kitzler, Hydrogen Migration and $\mathrm{C}-\mathrm{C}$ Bond Breaking in 1,3-Butadiene in Intense Laser Fields Studied by Coincidence Momentum Imaging, Chem. Phys. Lett. 484, 119 (2010).

[2] M. F. Kling, P. von den Hoff, I. Znakovskaya, and R. de Vivie-Riedle, (Sub-)Femtosecond Control of Molecular Reactions via Tailoring the Electric Field of Light, Phys. Chem. Chem. Phys. 15, 9448 (2013).

[3] M. Kremer, B. Fischer, B. Feuerstein, V. L. B. de Jesus, V. Sharma, C. Hofrichter, A. Rudenko, U. Thumm, C. D. Schröter, R. Moshammer, and J. Ullrich, Electron Localization in Molecular Fragmentation of $\mathrm{H}_{2}$ by Carrier-Envelope Phase Stabilized Laser Pulses, Phys. Rev. Lett. 103, 213003 (2009).

[4] Y. Liu, X. Liu, Y. Deng, C. Wu, H. Jiang, and Q. Gong, Selective Steering of Molecular Multiple Dissociative Channels with Strong Few-Cycle Laser Pulses, Phys. Rev. Lett. 106, 073004 (2011).

[5] T. Rathje, A. M. Sayler, S. Zeng, P. Wustelt, H. Figger, B. D. Esry, and G. G. Paulus, Coherent Control at Its Most Fundamental: Carrier-Envelope-Phase-Dependent Electron Localization in Photodissociation of a $\mathrm{H}_{2}^{+}$Molecular Ion Beam Target, Phys. Rev. Lett. 111, 093002 (2013).

[6] X. Xie, K. Doblhoff-Dier, S. Roither, M. S. Schöffler, D. Kartashov, H. Xu, T. Rathje, G. G. Paulus, A. Baltuška, S. Gräfe, and M. Kitzler, Attosecond-Recollision-Controlled Selective Fragmentation of Polyatomic Molecules, Phys. Rev. Lett. 109, 243001 (2012).

[7] A. E. Boguslavskiy, J. Mikosch, A. Gijsbertsen, M. Spanner, S. Patchkovskii, N. Gador, M. J. J. Vrakking, and A. Stolow The Multielectron Ionization Dynamics Underlying Attosecond Strong-Field Spectroscopies, Science 335, 1336 (2012).
[8] K. Hosaka, A. Yokoyama, K. Yamanouchi, and R. Itakura, Correlation Between a Photoelectron and a Fragment Ion in Dissociative Ionization of Ethanol in Intense NearInfrared Laser Fields, J. Chem. Phys. 138, 204301 (2013).

[9] M. Lezius, V. Blanchet, M. Y. Ivanov, and A. Stolow, Polyatomic Molecules in Strong Laser Fields: Nonadiabatic Multielectron Dynamics, J. Chem. Phys. 117, 1575 (2002).

[10] A. N. Markevitch, S. M. Smith, D. A. Romanov, H. B. Schlegel, M. Y. Ivanov, and R. J. Levis, Nonadiabatic Dynamics of Polyatomic Molecules and Ions in Strong Laser Fields, Phys. Rev. A 68, 011402(R) (2003).

[11] I. Znakovskaya, P. von den Hoff, S. Zherebtsov, A. Wirth, O. Herrwerth, M. J. J. Vrakking, R. de Vivie-Riedle, and M. F. Kling, Attosecond Control of Electron Dynamics in Carbon Monoxide, Phys. Rev. Lett. 103, 103002 (2009).

[12] Y. Mairesse, J. Higuet, N. Dudovich, D. Shafir, B. Fabre, E. Mével, E. Constant, S. Patchkovskii, Z. Walters, M. Y. Ivanov, and O. Smirnova, High Harmonic Spectroscopy of Multichannel Dynamics in Strong-Field Ionization, Phys. Rev. Lett. 104, 213601 (2010).

[13] H. W. van der Hart, Sequential Versus Non-Sequential Double Ionization in Strong Laser Fields, J. Phys. B 33, L699 (2000).

[14] V. L. B. de Jesus, B. Feuerstein, K. Zrost, D. Fischer, A. Rudenko, F. Afaneh, C. D. Schröter, R. Moshammer, and J. Ullrich, Atomic Structure Dependence of Nonsequential Double Ionization of $\mathrm{He}, \mathrm{Ne}$ and $\mathrm{Ar}$ in Strong Laser Pulses, J. Phys. B 37, L161 (2004).

[15] A. Rudenko, K. Zrost, B. Feuerstein, V. L. B. de Jesus, C. D. Schröter, R. Moshammer, and J. Ullrich, Correlated Multielectron Dynamics in Ultrafast Laser Pulse Interactions with Atoms, Phys. Rev. Lett. 93, 253001 (2004).

[16] L. Zhang, S. Roither, X. Xie, D. Kartashov, M. Schöffler, H. $\mathrm{Xu}$, A. Iwasaki, S. Gräfe, T. Okino, K. Yamanouchi, A. Baltuska, and M. Kitzler, Path-Selective Investigation of Intense Laser-Pulse-Induced Fragmentation Dynamics in Triply Charged 1,3-Butadiene, J. Phys. B 45, 085603 (2012).

[17] H. Xu, T. Okino, T. Kudou, K. Yamanouchi, S. Roither, M. Kitzler, A. Baltuska, and S.-L. Chin, Effect of Laser Parameters on Ultrafast Hydrogen Migration in Methanol Studied by Coincidence Momentum Imaging, J. Phys. Chem. A 116, 2686 (2012).

[18] S. M. Sharifi, A. Talebpour, and S. L. Chin, Double Ionization of Benzene Interacting with Strong Ti:Sapphire Laser Pulses, J. Phys. B 40, F259 (2007).

[19] S. M. Sharifi, A. Talebpour, and S. L. Chin, Double Ionization of Unsaturated Hydrocarbons Interacting with High-Power Femtosecond Laser Pulses, J. At. Mol. Opt. Phys. 2009, 1 (2009).

[20] C. Cornaggia and P. Hering, Nonsequential Double Ionization of Small Molecules Induced by a Femtosecond Laser Field, Phys. Rev. A 62, 023403 (2000).

[21] A. Hishikawa, H. Hasegawa, and K. Yamanouchi, Hydrogen Migration in Acetonitrile in Intense Laser Fields in Competition with Two-Body Coulomb Explosion, J. Electron Spectrosc. Relat. Phenom. 141, 195 (2004).

[22] M. Pitzer, M. Kunitski, A. S. Johnson, T. Jahnke, H. Sann, F. Sturm, L. P. H. Schmidt, H. Schmidt-Böcking, R. Dörner, 
J. Stohner, J. Kiedrowski, M. Reggelin, S. Marquardt, A. Schießer, R. Berger, and M. S. Schöffler, Direct Determination of Absolute Molecular Stereochemistry in Gas Phase by Coulomb Explosion Imaging, Science 341, 1096 (2013).

[23] A. Hishikawa, A. Matsuda, M. Fushitani, and E. J. Takahashi, Visualizing Recurrently Migrating Hydrogen in Acetylene Dication by Intense Ultrashort Laser Pulses, Phys. Rev. Lett. 99, 258302 (2007).

[24] T. Ikuta, K. Hosaka, H. Akagi, A. Yokoyama, K. Yamanouchi, F. Kannari, and R. Itakura, Separation of Ionization and Subsequent Electronic Excitation for Formation of Electronically Excited Ethanol Cation in Intense Laser Fields, J. Phys. B 44, 191002 (2011).

[25] S. Roither, X. Xie, D. Kartashov, L. Zhang, M. Schöffler, H. $\mathrm{Xu}$, A. Iwasaki, T. Okino, K. Yamanouchi, A. Baltuska, and M. Kitzler, High Energy Proton Ejection from Hydrocarbon Molecules Driven by Highly Efficient Field Ionization, Phys. Rev. Lett. 106, 163001 (2011).

[26] C. Cornaggia, M. Schmidt, and D. Normand, Laser-Induced Nuclear Motions in the Coulomb Explosion of $\mathrm{C}_{2} \mathrm{H}_{2}^{+}$Ions, Phys. Rev. A 51, 1431 (1995).

[27] S. Palaniyappan, R. Mitchell, N. Ekanayake, A. M. Watts, S. L. White, R. Sauer, L. E. Howard, M. Videtto, C. Mancuso, S. J. Wells, T. Stanev, B. L. Wen, M. F. Decamp, and B. C. Walker, Ionization of Ethane, Butane, and Octane in Strong Laser Fields, Phys. Rev. A 82, 043433 (2010).

[28] R. Dörner, V. Mergel, O. Jagutzki, L. Spielberger, J. Ullrich, R. Moshammer, and H. Schmidt-Böcking, Cold Target Recoil Ion Momentum Spectroscopy: A 'Momentum Microscope' to View Atomic Collision Dynamics, Phys. Rep. 330, 95 (2000).

[29] J. Ullrich, R. Moshammer, A. Dorn, R. Dörner, L. P. H. Schmidt, and H. Schmidt-Böcking, Recoil-Ion and Electron Momentum Spectroscopy: Reaction-Microscopes, Rep. Prog. Phys. 66, 1463 (2003).

[30] X. Xie, S. Roither, D. Kartashov, E. Persson, D. G. Arbó, L. Zhang, S. Gräfe, M. S. Schöffler, J. Burgdörfer, A. Baltuška, and M. Kitzler, Attosecond Probe of Valence-Electron Wave Packets by Subcycle Sculpted Laser Fields, Phys. Rev. Lett. 108, 193004 (2012).

[31] A. M. Sayler, T. Rathje, W. Müller, C. Kürbis, K. Rühle, G. Stibenz, and G. G. Paulus, Real-Time Pulse Length Measurement of Few-Cycle Laser Pulses Using Above-Threshold Ionization, Opt. Express 19, 4464 (2011).

[32] A. M. Sayler, T. Rathje, W. Müller, K. Rühle, R. Kienberger, and G. G. Paulus, Precise, Real-Time, Every-Single-Shot, Carrier-Envelope Phase Measurement of Ultrashort Laser Pulses, Opt. Lett. 36, 1 (2011).

[33] A. S. Alnaser, X. M. Tong, T. Osipov, S. Voss, C. M. Maharjan, B. Shan, Z. Chang, and C. L. Cocke, LaserPeak-Intensity Calibration Using Recoil-Ion Momentum Imaging, Phys. Rev. A 70, 023413 (2004).

[34] A. D. Yau, S. A. Perera, and R. J. Bartlett, Vertical Ionization Potentials of Ethylene: The Right Answer for the Right Reason?, Mol. Phys. 100, 835 (2002).

[35] E. Ohrendorf, H. Köppel, L. S. Cederbaum, F. Tarantelli, and A. Sgamellotti, Doubly Ionized States of Ethylene: Auger Spectrum, Potential Energy Surfaces and Nuclear Dynamics, J. Chem. Phys. 91, 1734 (1989).
[36] L. V. Keldysh, Ionization in the Field of a Strong Electromagnetic Wave, Zh. Eksp. Teor. Fiz. 47, 1945 (1964) [Sov. Phys. JETP 20, 1307 (1965)].

[37] M. W. Schmidt, K. K. Baldridge, J. A. Boatz, S. T. Elbert, M. S. Gordon, J. H. Jensen, S. Koseki, N. Matsunaga, K. A. Nguyen, S. Su, T. L. Windus, M. Dupuis, and J. A. Montgomery, Jr., General Atomic and Molecular Electronic Structure System, J. Comput. Chem. 14, 1347 (1993).

[38] C.-M. Liegener, Calculations on the Auger Spectra of Ethylene and Acetylene, Chem. Phys. 92, 97 (1985).

[39] T. Ibuki, T. Imamura, I. Koyano, T. Masuoka, and C. E. Brion, Dissociation of Doubly Charged $\mathrm{CH}_{2}=\mathrm{CD}_{2}$ and $\mathrm{CH}_{2}=\mathrm{CF}_{2}$ in the Region of Valence Shell Photoexcitation, J. Chem. Phys. 98, 2908 (1993).

[40] B. K. McFarland, J. P. Farrell, P. H. Bucksbaum, and M. Gühr, High Harmonic Generation from Multiple Orbitals in $\mathrm{N}_{2}$, Science 322, 1232 (2008).

[41] P. von den Hoff, I. Znakovskaya, S. Zherebtsov, M. F. Kling, and R. de Vivie-Riedle, Effects of Multi Orbital Contributions in the Angular-Dependent Ionization of Molecules in Intense Few-Cycle Laser Pulses, Appl. Phys. B 98, 659 (2010).

[42] H. Akagi, T. Otobe, A. Staudte, A. Shiner, F. Turner, R. Dörner, D. M. Villeneuve, and P. B. Corkum, Laser Tunnel Ionization from Multiple Orbitals in $\mathrm{HCl}$, Science 325, 1364 (2009).

[43] C. Wu, H. Zhang, H. Yang, Q. Gong, D. Song, and H. Su, Tunneling Ionization of Carbon Dioxide from Lower-Lying Orbitals, Phys. Rev. A 83, 033410 (2011).

[44] J. P. Farrell, S. Petretti, J. Förster, B. K. McFarland, L. S. Spector, Y. V. Vanne, P. Decleva, P. H. Bucksbaum, A. Saenz, and M. Gühr, Strong Field Ionization to Multiple Electronic States in Water, Phys. Rev. Lett. 107, 083001 (2011).

[45] X. M. Tong, Z. X. Zhao, and C. D. Lin, Theory of Molecular Tunneling Ionization, Phys. Rev. A 66, 033402 (2002).

[46] D. Pavičić, K. F. Lee, D. M. Rayner, P. B. Corkum, and D. M. Villeneuve, Direct Measurement of the Angular Dependence of Ionization for $\mathrm{N}_{2}, \mathrm{O}_{2}$, and $\mathrm{CO}_{2}$ in Intense Laser Fields, Phys. Rev. Lett. 98, 243001 (2007).

[47] K. Lammertsma, M. Barzaghi, G. A. Olah, J. A. Pople, A. J. Kos, and P.v. R. Schleyer, The Ethylene Dication: A Theoretical Study of The Ethylene Dication $\left(\mathrm{C}_{2} \mathrm{H}_{4}^{2+}\right)$ Potential-Energy Surface, J. Am. Chem. Soc. 105, 5252 (1983).

[48] X. M. Tong, Z. X. Zhao, A. S. Alnaser, S. Voss, C. L. Cocke, and C. D. Lin, Post Ionization Alignment of the Fragmentation of Molecules in an Ultrashort Intense Laser Field, J. Phys. B 38, 333 (2005).

[49] T. Zuo and A. D. Bandrauk, Charge-Resonance-Enhanced Ionization of Diatomic Molecular Ions by Intense Lasers, Phys. Rev. A 52, R2511 (1995).

[50] E. Lötstedt, T. Kato, and K. Yamanouchi, Enhanced Ionization of Acetylene in Intense Laser Fields, Phys. Rev. A 85, 041402 (2012).

[51] S. Bubin, M. Atkinson, K. Varga, X. Xie, S. Roither, D. Kartashov, A. Baltuška, and M. Kitzler, Strong Laser-PulseDriven Ionization and Coulomb Explosion of Hydrocarbon Molecules, Phys. Rev. A 86, 043407 (2012).

[52] X. Xie, S. Roither, M. Schöffler, S. Bubin, H. Xu, S. Erattuphuza, A. Iwasaki, K. Varga, D. Kartashov, G. Paulus, 
K. Yamanouchi, A. Baltuska, and M. Kitzler, Role of Proton Dynamics in Efficient Photoionization of Hydrocarbon Molecules, Phys. Rev. A 89, 023429 (2014).

[53] R. Torres, N. Kajumba, J. G. Underwood, J. S. Robinson, S. Baker, J. W. G. Tisch, R. de Nalda, W. A. Bryan, R. Velotta, C. Altucci, I. C.E. Turcu, and J.P. Marangos, Probing Orbital Structure of Polyatomic Molecules by High-Order Harmonic Generation, Phys. Rev. Lett. 98, 203007 (2007).

[54] C. I. Blaga, J. Xu, A. D. DiChiara, E. Sistrunk, K. Zhang, P. Agostini, T. A. Miller, L. F. DiMauro, and C. D. Lin, Imaging Ultrafast Molecular Dynamics with Laser-
Induced Electron Diffraction, Nature (London) 483, 194 (2012).

[55] L. Holmegaard, J. L. Hansen, L. Kalhøj, S. Louise Kragh, H. Stapelfeldt, F. Filsinger, J. Küpper, G. Meijer, D. Dimitrovski, M. Abu-samha, C. P. J. Martiny, and L. Bojer Madsen, Photoelectron Angular Distributions from Strong-Field Ionization of Oriented Molecules, Nat. Phys. 6, 428 (2010).

[56] J. L. Hansen, L. Holmegaard, J. H. Nielsen, H. Stapelfeldt, D. Dimitrovski, and L. B. Madsen, Orientation-Dependent Ionization Yields from Strong-Field Ionization of Fixed-inSpace Linear and Asymmetric Top Molecules, J. Phys. B 45, 015101 (2012). 